\title{
A Study on Ubiquitous Environments Based on the Animal and Plant Management System in Green House
}

\author{
Min-Nyeon Kim ${ }^{1} \cdot$ Soon-Hwa Baek ${ }^{1}$
}

Published online: 14 February 2017

(C) The Author(s) 2017. This article is published with open access at Springerlink.com

\begin{abstract}
In this paper, we develop ubiquitous sensor network system that send alarm massage to users having portable devices such as mobile smart phone and its messages to detect the growth of such plants in the state farms in green house. As well, we develop the windows environment programs that can directly control the configuration environment through computer themselves from the farmers.
\end{abstract}

Keywords Ubiquitous sensor network - Cellular network - Animal and plant status · Portal handheld devices

\section{Introduction}

Current rural areas are suffering from labor shortages. For that reason, automatic information delivery system is urgently needed in order to compensate for the lack of labor. In this paper, we develop the system detect the state of the growth of farm animals and plants and inform portable devices such as smart phones etc. Additionally, we develop the windows environment programs that can control the configuration environment through your computer directly from the farmers in Fig. 1 [1-4].

In this paper, we have developed the system which transmit information of porcine temperature and methane concentration, humidity sensor under the green house to the user.

According to the known, pig's body temperature changes affect the rate of pregnancy. As it detects a change more than 38 degree temperature of the pig, the farmer recognize optimum time of piggy insemination. Therefore, it is so important that inform the farmer to

Min-Nyeon Kim

myki@bu.ac.kr

Soon-Hwa Baek

baek@bu.ac.kr

1 Division of Information and Communication Engineering, Baekseok University, Cheonan 330-704,

Korea 

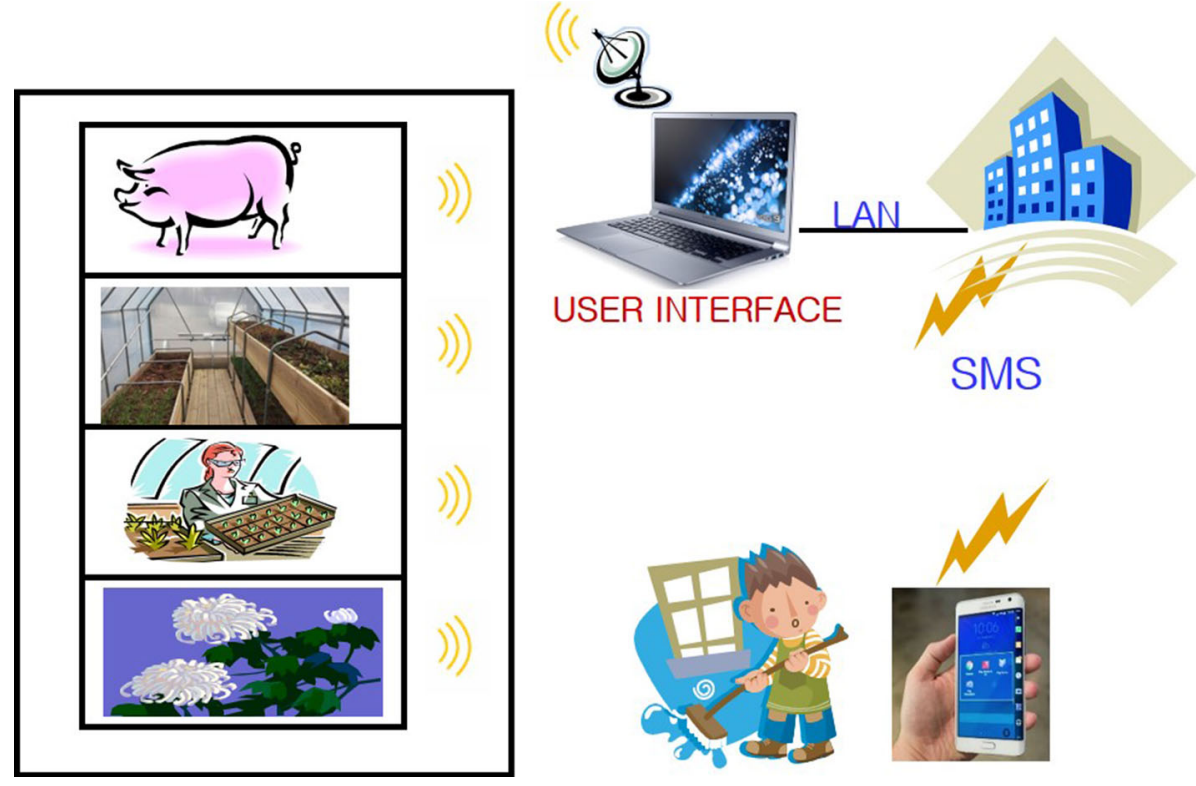

Fig. 1 Management system diagram of animal and plant life

detect porcines condition by measuring piggy temperature change. Also, In a timely, the system can contribute to farmer's work informing the water state (Prevention excessive humidity), temperature, carbon dioxide, methane concentration in green house, etc. For example, the system can be tell to users the humidity if there is not the appropriate status of crop in green house. Methane is needed but too much inhibits plant growth. The information of all can be modified appropriate value of smart phone by users.

Also, for user who do not use the smart phone, is suppose to develop an easy-to-use Windows environment. The all of these services require the server that can services the $3 \mathrm{G}$, LTE-network, USN.

In the same farmhouse of the above management systems and plants are installed should be able to apply to himself the status change to the Windows environment. For example, the environmental interface must be implemented in a mobile phone number, changing of animals and plants to be easily adjusted on the computer in Fig. 2.

Users of farmer to easy adapt to the environment in which the display interface according to the type of sensor to the configuration so that you can easily make changes in the Windows environment when the body temperature is different from the normal temperature corrected timely and if the dangerous condition placed to set the humidity and methane amounts to prevent over hydration in greenhouses to carry a mobile phone [4-10].

Data origin for existing users 3G, LTE to taken to be network server installation and operation of farms in order to integrate with them is essential. Farm supply of server environments easily in conjunction with cell phone use was made easier to use and is intended.

In this paper, sensor node CC2430 is achieved by the actions as TinyOS and configuration within the program is language using a miniature $\mathrm{NesC}$ programming. 


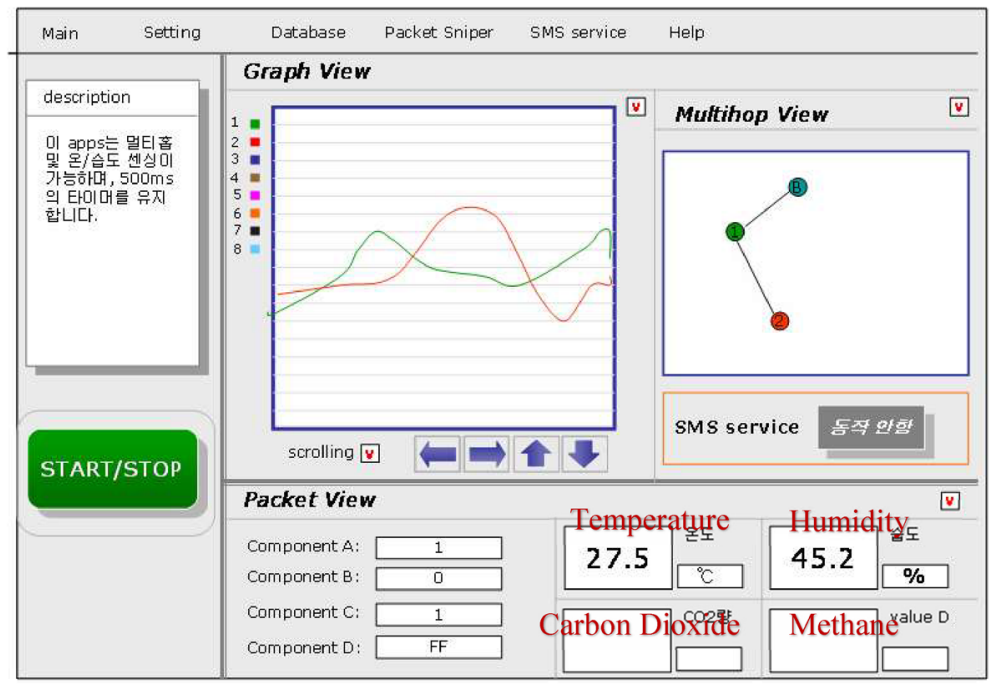

Fig. 2 User interface environment

\section{Research Content}

\subsection{Background Theory}

In this paper, we mainly have purposes of control various values purposes to control pig pregnancy rates, methane quantity, $\mathrm{CO}_{2}$ concentration and brightness. These factors have a big impact on the crops.

\subsubsection{Optimum Time of Insemination for Pig}

Reportedly, after the body temperature of the pig reach $38^{\circ}$, its optimum time of insemination has been less for 2 days such as Fig. 3. At the time of raising the body temperature of pigs between 24 and $36 \mathrm{~h}$ is the most appropriate insemination time. The labor force is the appropriate time to go beyond just the lack of a farmhouse apt to give an appropriate notification in advance.

\subsubsection{Carbon Dioxide Concentration and Humidity in the Green House}

Meanwhile, in green houses, carbon dioxide is great harm to humans and crops as it reach more than a certain amount. But it is essential for it to produce a crop fertilizer supply process. Thus, using a USN sensor can be maximized to protect the human health and the crop revenues if controlling the amount of methane.

In addition, it is important to control the amount of moisture in the greenhouse. The humidity increases when the temperature difference between inside and outside the house heavily. When the humidity is high, pest occurs well. 


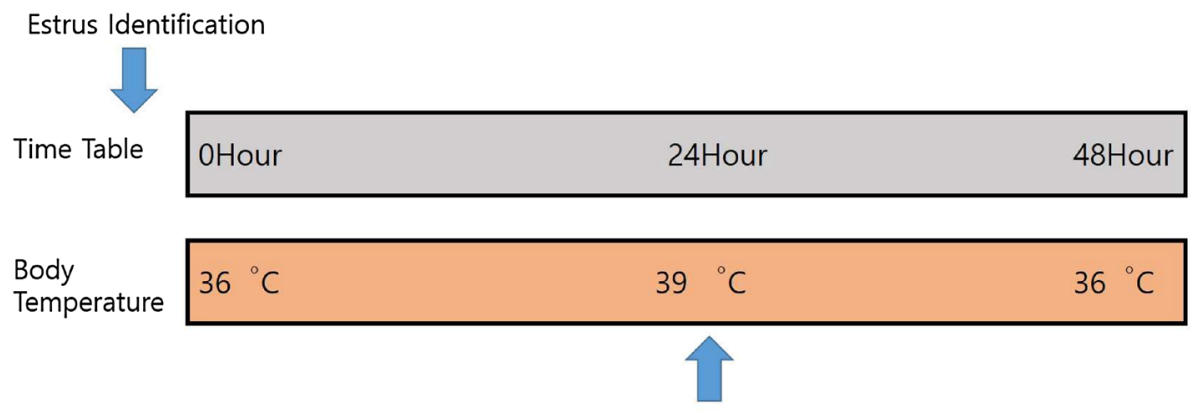

Optimum time of Insemination

Fig. 3 Optimum time of insemination

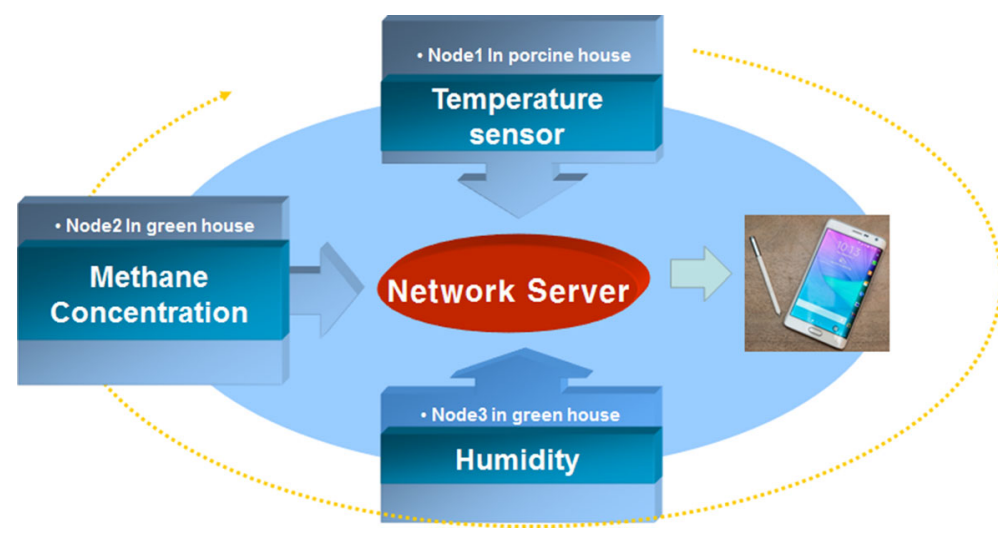

Fig. 4 System configuration

\subsection{Application Theory}

It is configured to operate with the system of Fig. 4. USN sensor of small forwards environmental conditions such as temperature, humidity as text messages to smart phones of managers and administrators can modify through their computer environment parameters [4].

The data indicates that the oscillation sensor data acquisition devices to users via SMS transfer wireless module. If the notification process is dangerous and one has to receive regular intervals if the modification is one less outgoing messages after a certain period of time (about 12-24 h) with prior notice.

Sensor data generating apparatus has a built-in battery, and each sends the detected data to the CPU operation has itself to the wireless module in Fig. 5.

A wireless module to the configuration through the PC, the user need. This assignment makes it possible to work with the hardware of the wireless module using a visual program in Fig. 6.

\subsection{Hardware and Software Presentation}

The hardware used in this task by using the CC2430 chip was chipcon's firmware using the 8051 program. Was linked to the windows and use the TinyOS 2.0 environment, user 


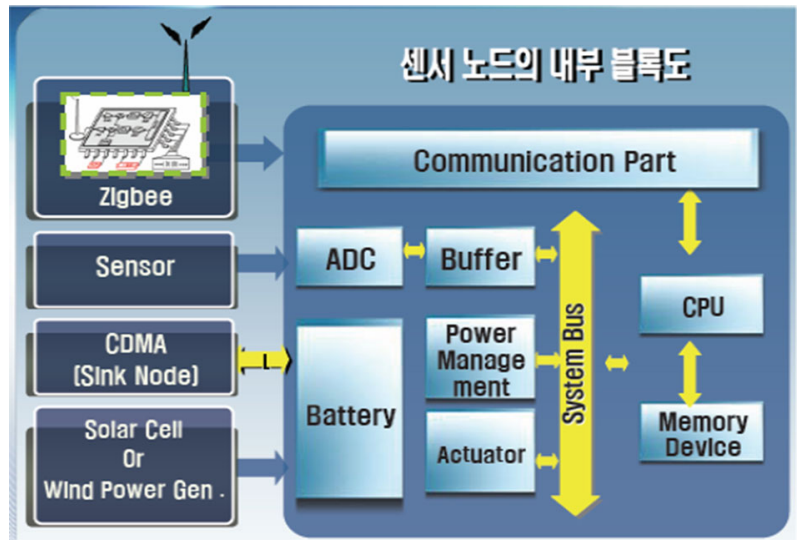

Fig. 5 Sensor data generator in each node
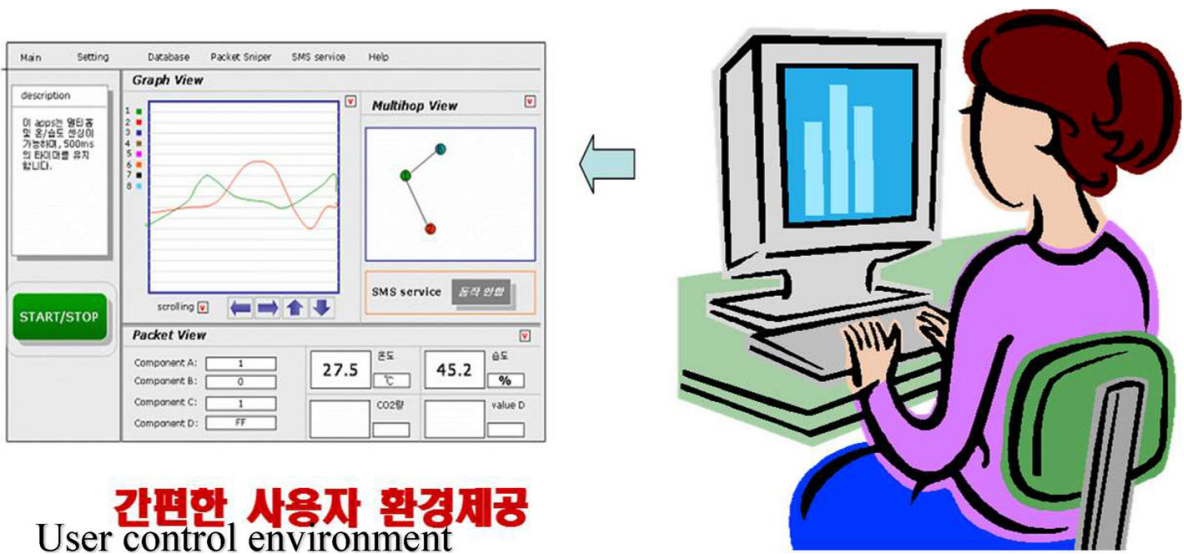

Fig. 6 Graphical user interface service

interface was programmed using Visual $\mathrm{C}++$ 6.0. Environment for the operation is shown below (Table 1).

\subsubsection{Programming}

USN built the CC2430 chip is programmed using the NesC under TinyOS 2.0. The main programming information, the operation has a low-power stop mode to operate an event occurs environments. The main program, such as setting time is shown below. 
Table 1 Hardware spec and used software

\begin{tabular}{ll}
\hline Service environment & Architecture \\
\hline 0.1 ODBC 3.0 & Decom SMS Client215 \\
0.2 Pentium IV & MS-SQL \\
0.3 Memory $512 \mathrm{MB}$ 이상 & Fauna status notification (application) TinyOS-2 \\
\hline
\end{tabular}

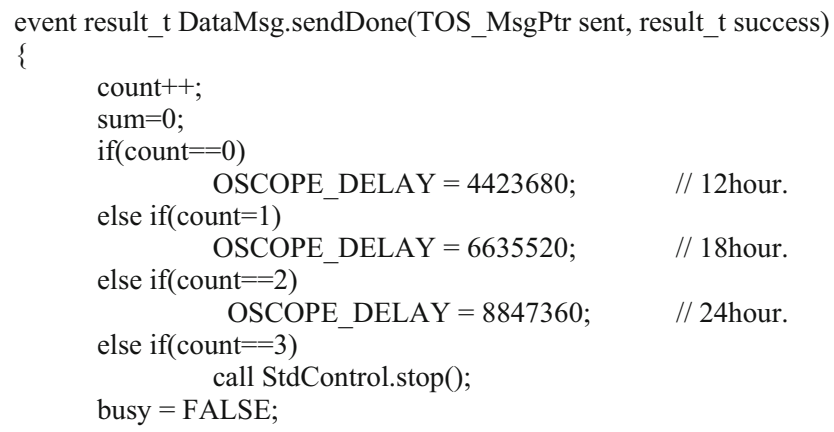

\subsubsection{P.C Server Setting}

After the user has installed on your PC, MS-SQL and ODBC to register. To send a text message to the user's hand port after installing the program that the company's SMS transmission service should be linked to the server. 


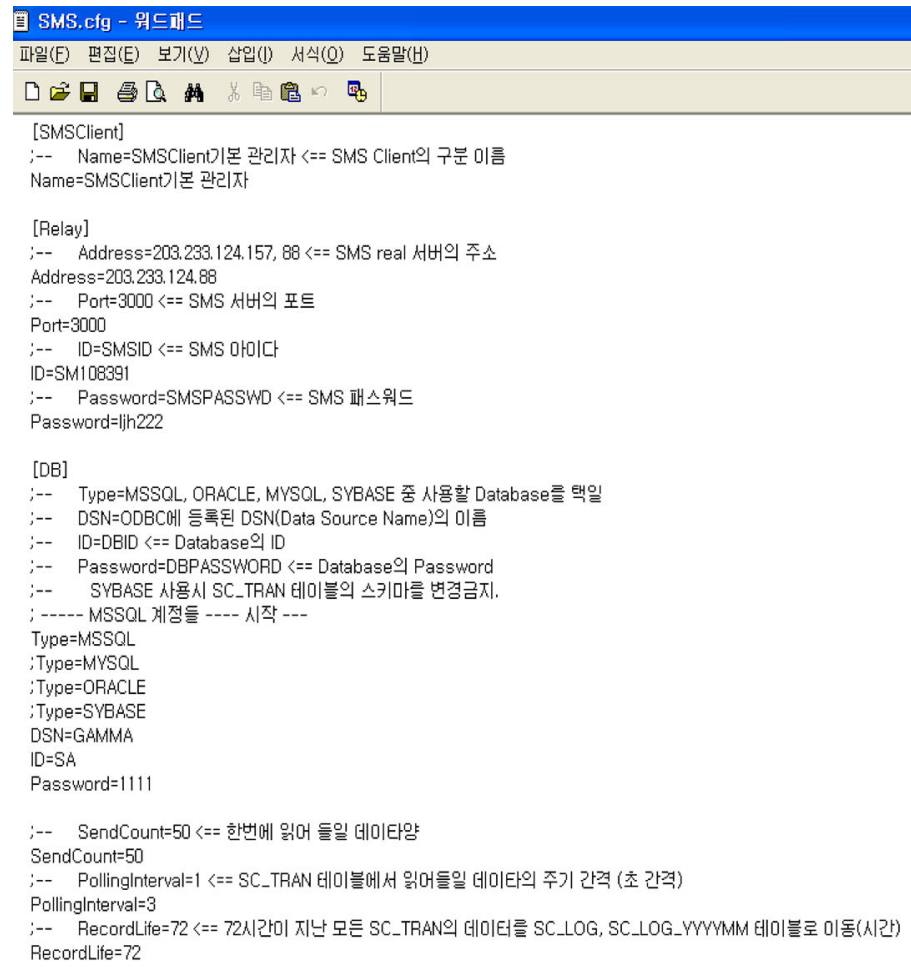

\section{ID $=$ SM108391}

\section{:-- Password=SMSPASSWD <== SMS 패스워드}

Password=lin222

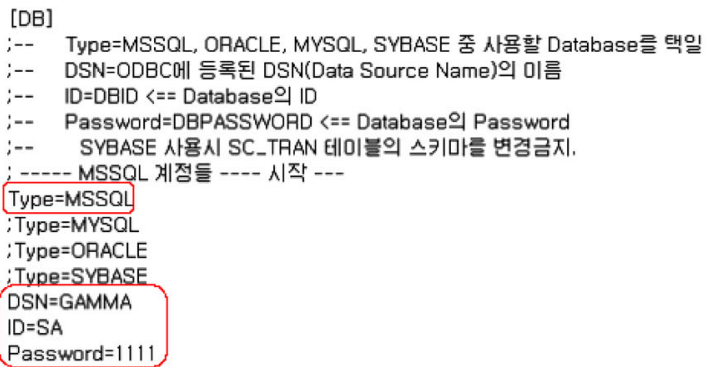

\subsubsection{User Interface Program}

The user interface program was written in Visual $\mathrm{C}++$.

In the screen of Fig. 7 above shows that by setting the user's telephone number, reminder number (USN sensor), etc. to receive the transmitted temperature, humidity, $\mathrm{CO}_{2}$ concentration and the like. 


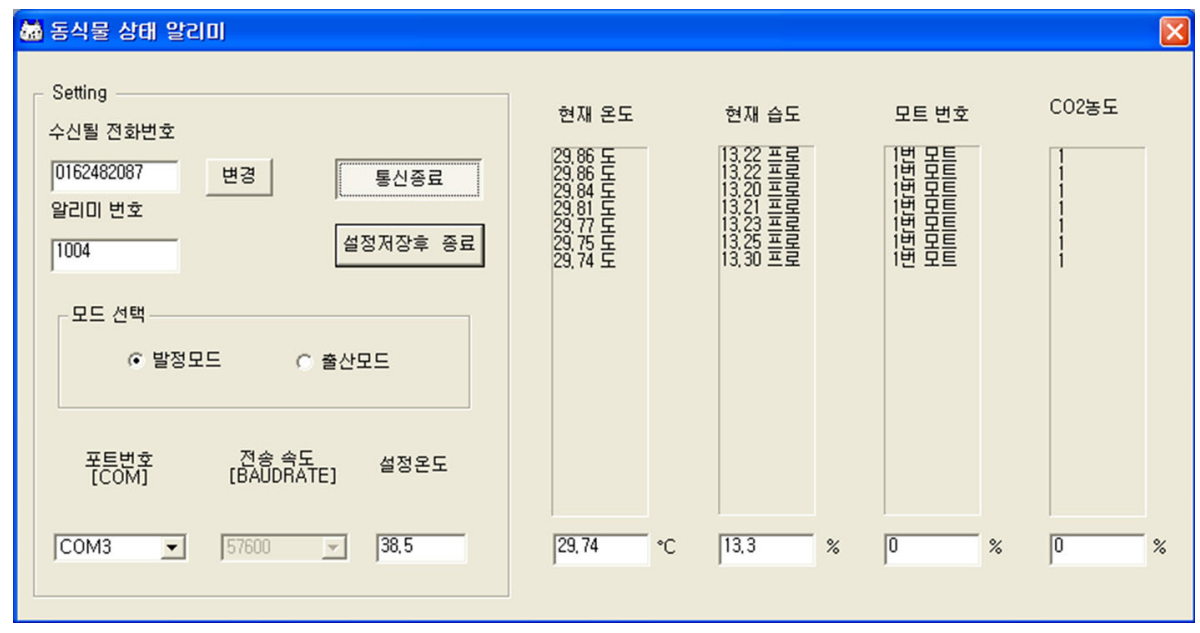

Fig. 7 Setting environments and results
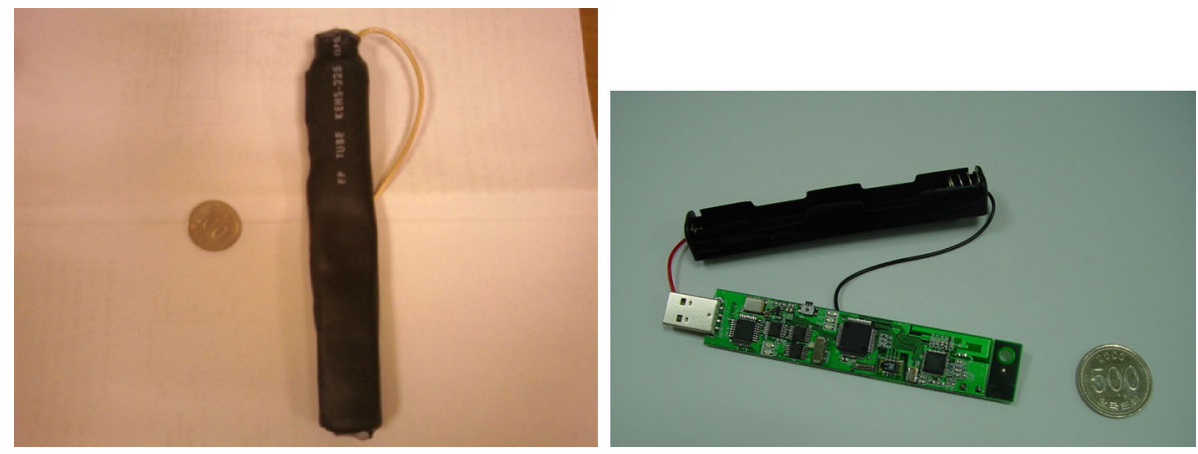

Fig. 8 Producted USN sensor for insertion (uterine)

\section{Conclusions}

In this paper, three outcomes in focusing on research results. The first is pig skin temperature changes and correct at the time to inform users. The second and third are results whose the methane and humidity to inform users within green house. In front of the hardware and software produced results show by dividing as follows.

\subsection{Production Results}

In Fig. 8, it producted USN sensor of Node1 for insertion of a breeding pig body (in uterine) that is relatively larger than normal it. This product contains the battery, but low power consumption as well send to user message below certain value.

In Fig. 9, it is intended to test Node1 of USN sensor for porcine temperature as the device of incubator for a thermo-hydrostat. The user can program it so that is to remain and have at times such as high temperature in porcine body. 
Fig. 9 Incubator for a thermohygrostat

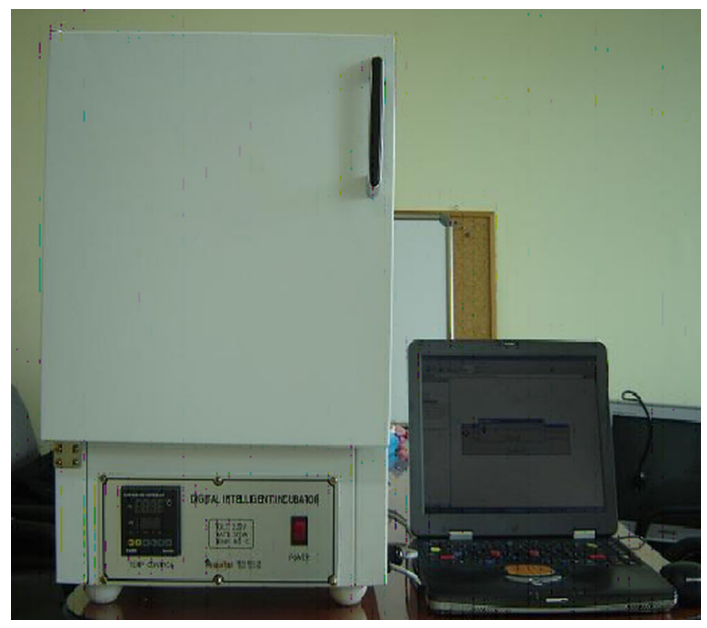

Fig. 10 USN sensor for humidity, methane and temperature
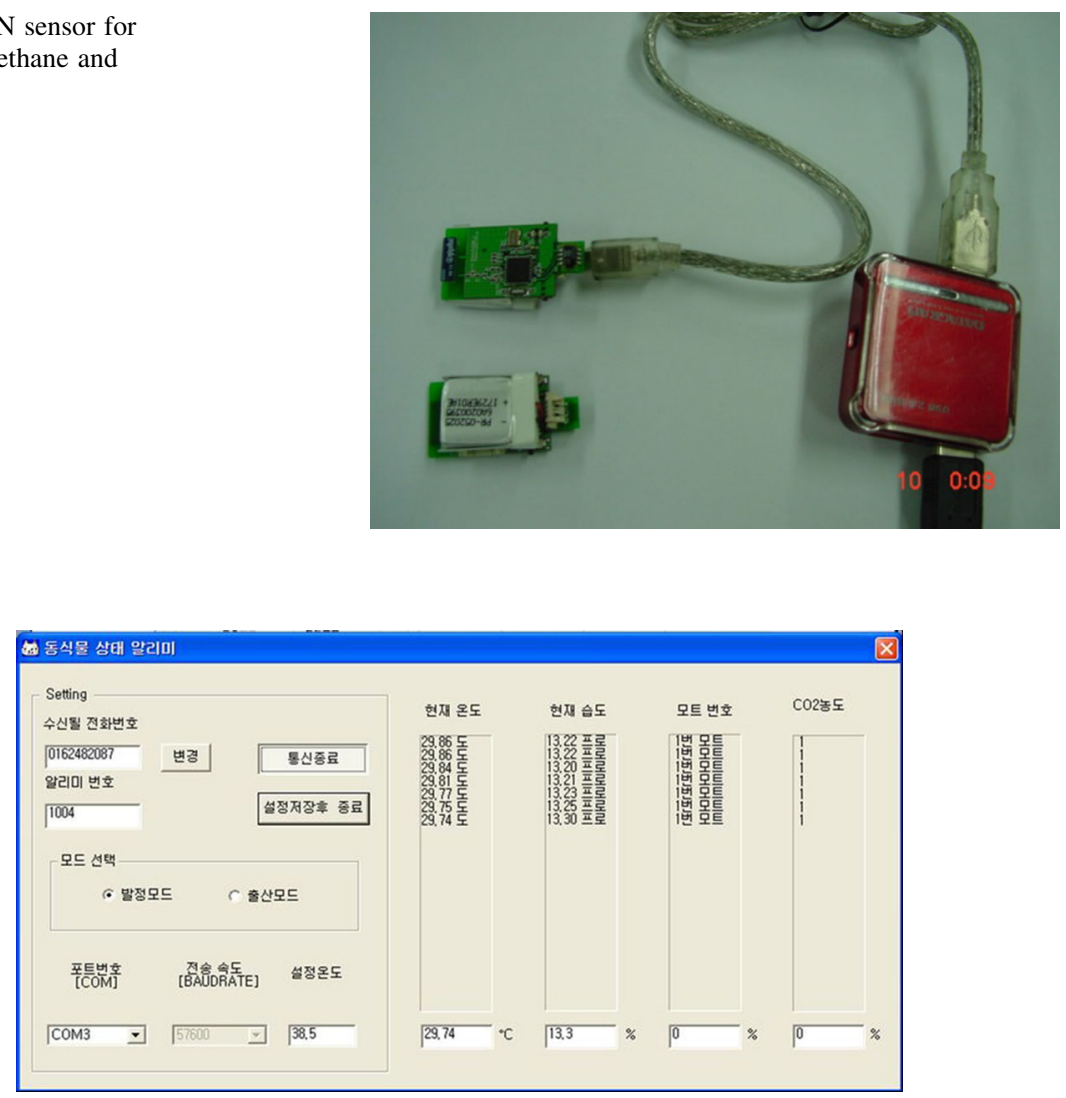

Fig. 11 Display SMS for a alarm of each states 
In addition, the USN sensor of Fig. 10 is constructed sensor to measure humidity, temperature and methane concentration. In Fig. 11 GUI (Graphical User Interface) is display SMS for an alarm of each state.

\subsection{Discussion}

Until now, we have used usn properly in the farmhouse. In this paper, there are two other topics. How to appropriately convey the environment of the greenhouse to the farmers. Both can be solved in smart networks such as USN. In the future, we expect the future to be used more conveniently by the usn and others.

This may be referred to a task irregularly bonded junction the field of bio-technology to ubiquitous sensor networks. In particular, ubiquitous sensor can be referred to an important issue that is currently receiving attention are combined with biotechnology can greatly be called encouraging.

This approach can be seen as a technical challenge has been downsized due to the development of the new OS developed lightweight and mobile transceiver especially works of $3 \mathrm{G}$, LTE could give birth to a very successful outcome. It is expected to develop through continuous development.

Open Access This article is distributed under the terms of the Creative Commons Attribution 4.0 International License (http://creativecommons.org/licenses/by/4.0/), which permits unrestricted use, distribution, and reproduction in any medium, provided you give appropriate credit to the original author(s) and the source, provide a link to the Creative Commons license, and indicate if changes were made.

\section{References}

1. Romer, K., \& Mattern, F. (2004). The design space of wireless sensor network. IEEE Wireless Communications, 11(6), 54-61.

2. Ryu, D. H. (2013). A development of urban farm management system based on USN. The Journal of the Korea Institute of Electronic Communication Sciences, 8(12), 1917-1922.

3. Telecommunications Technology Association. (2013). Greenhouse control system-part 4: Interface standard between greenhouse operating system and integrated greenhouse management system. TTAK. K O -06.02 88 -Part4.

4. Ministry of Agriculture, Food and Rural Affairs. (2013). Spreading plan for ICT convergence of agricultural food (Vol. 8).

5. Kim, M.-Y., Seo, D.-J., Byun, J.-B., \& Kang, J.-K. (2015). ICT-based living in the contact type service model for self-life support of the elderly living alone. Journal of Digital Convergence, 13(4), 25-38.

6. Akyildiz, I. F. (2002). Wireless sensor networks: A survey. Computer Networks, 38(4), 393-422.

7. Kim, Y.-T., \& Jeong, Y.-S. (2015). Optimization routing protocol based on the location, and distance information of sensor nodes. Journal of Digital Convergence, 13(2), 127-133.

8. Lee, Y.-H., Cho, H.-J., \& Lee, J.-H. (2015). Implementation of educational service for environmental saver using smart device. Journal of Digital Convergence, 13(5), 1-8.

9. Jung, J.-W., Kim, J., Song, M.-G., \& Jin, C.-G. (2015). A study on development of certification schemes for cloud security. Journal of Digital Convergence, 13(8), 43-49.

10. Jung, H.-J. (2015). The analysis of data on the basis of software test data. Journal of Digital Convergence, 13(10), 1-7. 


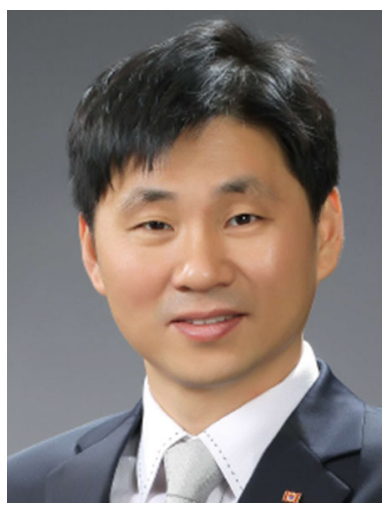

Min-Nyeon Kim received the Ph.D. degree in Electronic Engineering from Hong-Ik University, Korea, in 2003. He is currently a full time professor of Baek-seok University cheonan Korea. He had been researcher at DAEWOO Electronics, Korea in 1995-1998 and conduct project of anti-radar missile in 2005-2007. He is primarily involved in radar related projects. His current field of research is automotive radar.

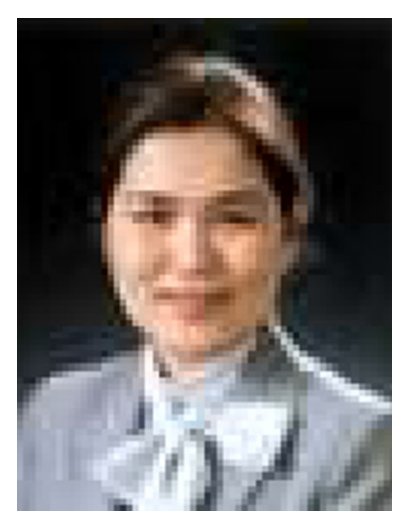

Soon-Hwa Baek received the Ph.D. degree in Computer Science from Chung-buk national University, Korea, in 2000. She is currently a full time professor of Baek seok University cheonan Korea. She has been Visiting Researcher in University of Maryland, CFAR in 1997.. Her current field is development of Building of monitoring and smart system on the companion animal delivery management using Bio sensing Using Real-time Monitoring. 